\title{
SPREAD OF PSEUDOMONAS AERUGINOSA IN A BURNS UNIT
}

\author{
S.-O. Liljedahl*, ANNa-Stina Malmborg $\dagger$, B. Nyström $\dagger$ and L. SJöberg $\ddagger$ \\ Departments of * Surgery and $\dagger$ Clinical Microbiology, Karolinska Hospital, \\ and $\ddagger$ National Bacteriological Laboratory, Stockholm, Sweden
}

IN recent years, severe infections with Pseudomonas aeruginosa, which are often fatal, have become increasingly common in patients with burns (Moncrief and Teplitz, 1964; MacMillan, 1965; Altemeier, Todd and Inge, 1966; Müller, 1967). This organism is often acquired in the burns units of hospitals (Lowbury and Fox, 1954; Sutter and Hurst, 1966; Barclay and Dexter, 1968; Lowbury, 1970). Studies of the spread of $P$. aeruginos $a$ have been greatly facilitated since, among other things, the advent of the phage-typing technique (Graber et al., 1962; Lindberg et al., 1964; Sutter, Hurst and Fennell, 1965; Sjöberg and Lindberg, 1968).

Since 1954, no increase in the incidence of clinically manifest pseudomonas infections has been observed in the Burns Unit of the Karolinska Hospital (Birke, Liljedahl and Norberg, 1969; Wickman, 1970). The frequency of colonisation with $P$. aeruginosa in extensive burns has also remained constant at about 50 per cent. Since colonisation with pseudomonas did not occur often until after some weeks in hospital, these authors concluded that these bacteria were mainly acquired by cross-infection in the hospital.

We now report an epidemiological study of $P$. aeruginosa in this Unit carried out with the help of the phage-typing technique.

\section{MATERIALS AND METHODS}

\section{The Burns Unit}

The Unit at the Karolinska Hospital serves as a special clinic for burns, mainly of a severe nature, from various parts of Sweden. It has seven single rooms, each with an anteroom. It also includes a small operating theatre, a bathroom, an examination room, a sluice room and a nurses' office. In each ante-room there is a wash-basin, where everybody entering or leaving the room must wash and disinfect the hands. There is also a sink, and a container with a phenolic disinfectant for the disinfection of all non-disposable material that has been in contact with the patient. Non-disposable bed-pans and urinals are, however, disinfected by hot water in the sluice-room. In the ante-room are placed gowns and disposable gloves, that everybody entering the room for treatment or nursing of the patient has to put on. Clean gowns are provided daily. There is also a textile bag for used linen and a receiver lined with a disposable plastic bag for used disposables. The Unit is not ventilated in such a way as to prevent air movement between rooms. All skin transplants are made in the operating theatre.

\section{The patients}

Seventy-three patients were studied; their age distribution is shown in table I. As in other reports from the Unit (e.g., Birke et al., 1964), burns were classified in three groups as

Received 26 Feb. 1972; accepted 16 Apr. 1972.

J. MED. MICROBIOL.-VOL. 5 (1972) 
follows: group I are small burns of total area up to 15 per cent. and with third-degree burns up to 5 per cent. of the body surface; group II are medium-sized burns of total area 15-30 per cent. and with third-degree burns of 5-15 per cent. of the body surface; group III are extensive burns of total area exceeding 30 per cent. and with third-degree burns of over 15 per cent. of the body surface. In addition, nine patients without burns but with extensive and deep skin lesions were admitted to the Unit during this investigation.

The methods of treating burns in the Unit have been described by Barr et al. (1967), Liljedahl (1970) and Wickman. All the burns are treated by exposure. No excisions are made earlier than $2 \mathrm{wk}$ after the injury. After the $2 \mathrm{nd}$ wk the patients are bathed two or three times weekly, at which time necrotic eschars are carefully removed. No topical treatment is given. Major burns are treated with warm, dry air in special beds provided in three of the rooms. To make optimal use of these beds, the patients had often to be moved from one room to another.

\section{Bacteriological methods}

Samples for culture were collected from the burns with a sterile swab on admission and thereafter at least once a week. During the later part of the study faecal samples were

TABLE I

Age distribution of the patients studied

\begin{tabular}{cccccccccc|c}
\hline \multicolumn{1}{c}{ Number of patients of age (yr) } \\
\hline $0-9$ & $10-19$ & $20-29$ & $30-39$ & $40-49$ & $50-59$ & $60-69$ & $70-79$ & $80-89$ & $90-$ & any \\
\hline 2 & 6 & 12 & 9 & 11 & 9 & 15 & 6 & 2 & 1 & 73 \\
\hline
\end{tabular}

collected from all patients once a week. When infection was suspected, samples were collected also of blood, urine and tracheal secretion.

Samples were collected with a sterile swab from wash-basins and sinks in all patient and service-rooms. This was done by a uniform method and from the same sampling points on all occasions. During the first months of the study these environmental samples were collected only sporadically, during wk 20-35 regularly once a week, and thereafter once a month. On a few occasions, in relation to patients with severe pseudomonas colonisation, samples were collected also from flower-vase water, drinking glasses, door-handles, etc. All cultures were seeded in the microbiological laboratory of the hospital within $1 \mathrm{hr}$ of sampling.

All samples, except of faeces, were incubated aerobically and anaerobically according to the laboratory routine. The faecal samples were cultured on cetrimide agar and in tetrathionate broth. All morphologically distinguishable colonies from the same culture were subcultured to new plates for verification and typing. Bacteria were usually identified as $P$. aeruginosa if they produced a bluish-green pigment on King's medium (King, Ward and Raney, 1954).

Phage typing. The typing was done on Trypticase Soy Agar (Baltimore Biological Laboratories) flooded with a growing bacterial culture. The 18 phages were applied at the routine test dilution $($ RTD) and at 100 times this strength $(\mathrm{RTD} \times 100)$. Lysis was recorded after incubation at $30^{\circ} \mathrm{C}$ overnight according to Sjöberg and Lindberg.

Disinfection of wash-basins and sinks. Attempts were made to remove pseudomonas from wash-basins and sinks with Sanivit (Jeyes Sanitary Compounds Co. Ltd, England), a detergent that has a bactericidal effect by releasing $50 \mathrm{mg}$ of free chlorine per 1 .

In a laboratory experiment, a wash-basin contaminated with $P$. aeruginosa was cleaned with Sanivit, which was also poured into the outlet of the wash-basin. Samples for culture 
were collected 1, 2, 3, 4 and $6 \mathrm{hr}$ after this procedure, the wash-basin not being used in the meantime. In another otherwise similar experiment the wash-basin was flushed for 5 min. per hr during the entire period of the experiment. Cleaning of wash-basins and slop-basins with Sanivit was thereafter introduced in the Unit as part of the daily routine. Samples for culture were collected before and $5 \mathrm{hr}$ after this cleaning.

\section{RESULTS \\ Isolation and phage typing of Pseudomonas aeruginosa}

Excluding faecal cultures, $P$. aeruginosa was isolated on one or more occasions from 45 of the 73 patients, from 23 of 29 in burn-group III, 11 of 20

TABLE II

Phage typing of Pseudomonas aeruginosa cultures isolated from the burns unit

\begin{tabular}{|c|c|}
\hline Phage-typing pattern & $\begin{array}{c}\text { Number (and percentage) } \\
\text { of isolates }\end{array}$ \\
\hline $\begin{array}{l}21 / 1214 / 68 / 109 / \mathrm{F} 8 \\
21 / 44 / 1214 / 68 / 109 / \mathrm{F} 8 \\
1214 / 68 / 109 / \mathrm{F} 8 \\
21 / 1214 / 109 / \mathrm{F} 8 \\
1214 / 109 / \mathrm{F} 8 \\
\text { Total "strain A "* }\end{array}$ & $\begin{array}{r}111 \\
41 \\
19 \\
13 \\
13\end{array}$ \\
\hline $\begin{array}{l}120 \text { other patterns } \\
\text { Not typable }\end{array}$ & $\begin{array}{r}394(65) \\
14(2)\end{array}$ \\
\hline All strains & 605 \\
\hline
\end{tabular}

in group II, 4 of 15 in group I, and from 7 of 9 patients with other skin lesions. In all it was isolated from skin lesions in 39 patients, from urine in five, from tracheal secretion in one, from sputum in one and at autopsy in one patient. There was no correlation between the age of the patients and the frequency of isolation of $P$. aeruginosa. Regular weekly cultures were made of the faeces of 33 patients. In 17 of them (52 per cent.) $P$. aeruginosa was isolated on at least one occasion. In 11 of the 17 patients it was found also in the burn. Eight of them carried it in the burn before it was found in the faecal flora. Three first had pseudomonas in the burn after or simultaneously with its appearance in the faeces.

In all, $P$. aeruginosa was isolated on 605 occasions, on 194 from patients and on 411 from the environment. On phage typing, 125 different patterns of lysis were obtained. Five of these patterns were assigned to a main phage pattern which always included lysis by phages 1214, 109 and F8 and which sometimes included lysis by one or more of phages 21,44 and 68 (table II). Thirty-three per cent. of the isolates were assigned to this main phage pattern. These we called "strain A". This strain was isolated from 21 of the 45 
colonised patients. Of the 17 patients with $P$. aeruginosa in their faeces six carried strain A. Only 14 isolates ( 2 per cent.) were untypable with the set of phages used. There was no statistically significant difference between isolates from patients and from the environment in the frequency of strain A.

\section{Clinical infections}

Although $P$. aeruginosa was isolated in 45 of 73 patients, there were few manifest infections. Two-both due to strain A-were of a serious nature, and one had a fatal outcome.

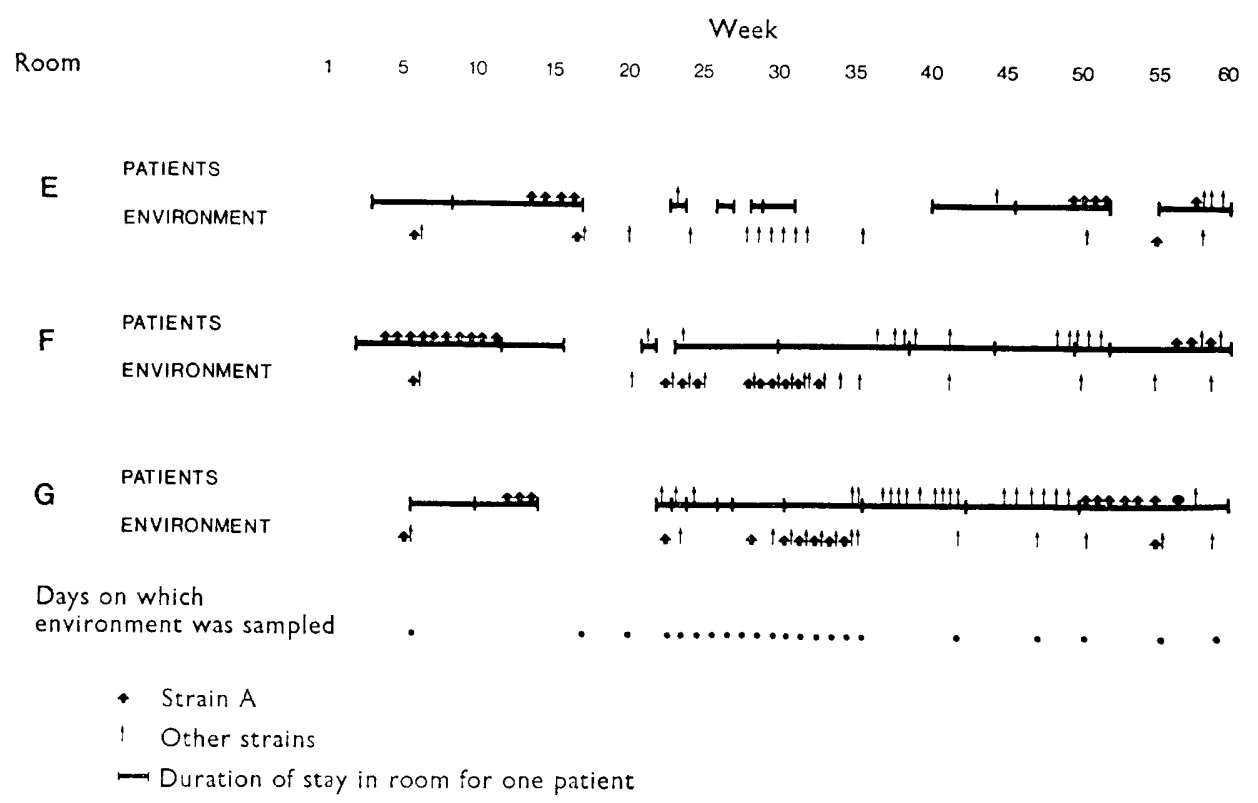

FIG. 1.-Isolation of Pseudomonas aeruginosa from patients and environment in rooms with warmair beds.

\section{Epidemiology}

Rooms with warm-air beds. At the start of the study, strain A was isolated in the environment in all three rooms, but only the patient in room F was colonised with the strain (fig. 1). Later, patients in the other rooms became colonised with the same strain. Other pseudomonas strains were encountered in the environment but not in the patients. During wk 17-21, in the summer, the Unit was closed and was thoroughly cleaned. Strain A was nevertheless found in the environment in two of the rooms after the clean-up but before the Unit was reopened, and other pseudomonas strains were isolated at the same time. Although strain A was present in the environment for more than $10 \mathrm{wk}$ after the ward was reopened it did not appear in any patients. Then it disappeared also from the environment. It re-occurred in one patient in wk 49 and $1 \mathrm{wk}$ later in another patient. Not until 6 wk later did it reappear in the environment in the rooms in which these patients were being treated. 
Rooms without warm-air beds. In room A, strain A was isolated both in the environment and from most patients during the entire period of the study (fig. 2). In rooms $B$ and $C$ the strain was isolated only sporadically both from patients and from the environment during the entire period of the study. In room D the strain was isolated in the environment during the entire period of the study, but after the summer closure only on one occasion from one patient.

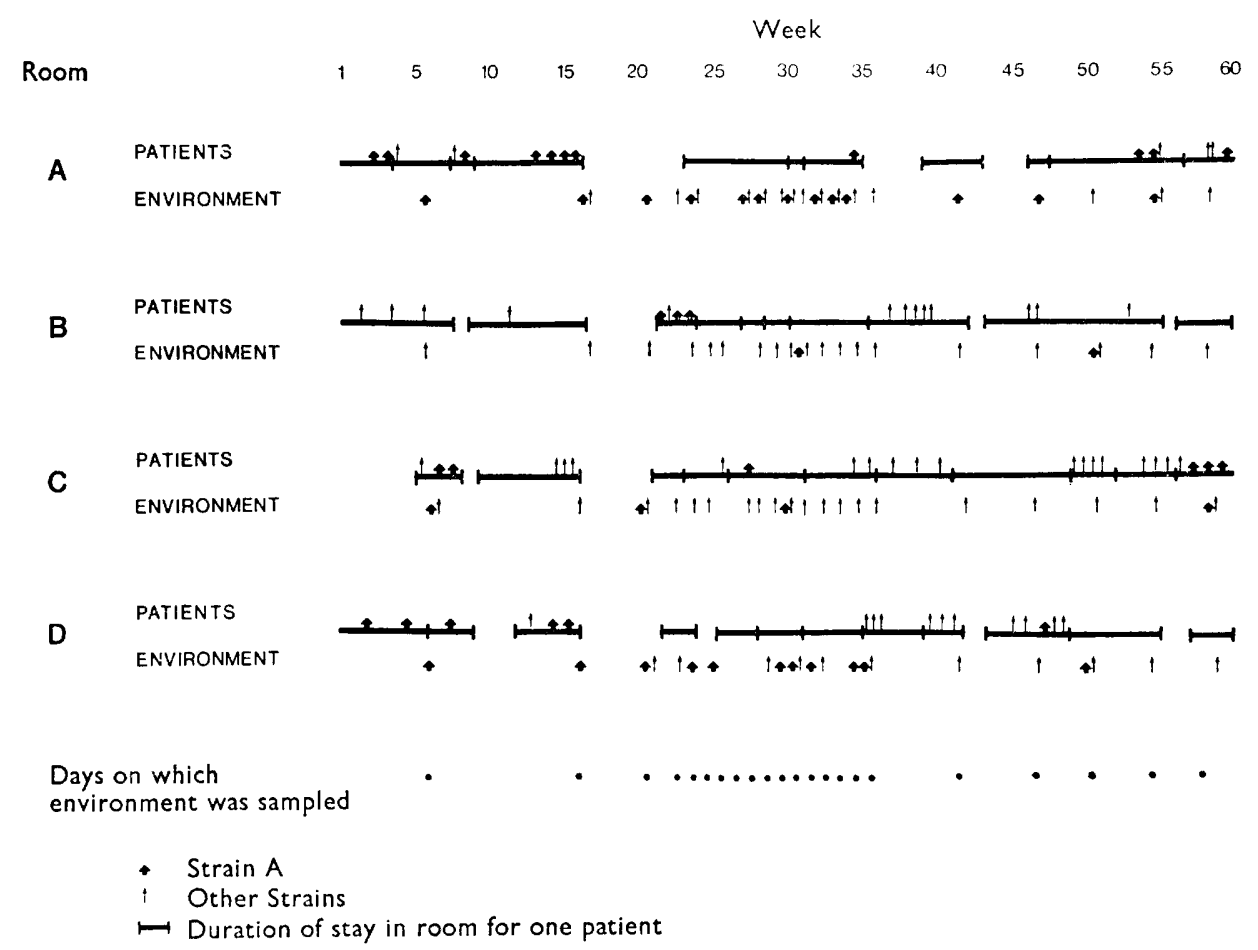

FIG. 2.-Isolation of Pseudomonas aeruginosa from patients and environment in rooms without warm-air beds.

In all rooms with and without warm-air bed pseudomonas strains other than strain A were isolated from the environment as well as from patients during the entire period of the study.

Other rooms. As will be seen from fig. 3, strain A was isolated throughout the period of the study from the operating theatre, the examination room and the sluice room. It was never discovered in the nurses' office, where, on the other hand, another pseudomonas strain was regularly found. In the bathroom strain A was isolated only once.

\section{Disinfection of wash-basins}

The laboratory experiment showed a reduction in number of $P$. aeruginosa immediately after disinfection of the wash-basin. After $6 \mathrm{hr}$, however, the number of bacteria had returned to the initial value even if the wash-basin was not used in the meantime (fig. 4). 
478 S.-O. LILJEDAHL, A.-S. MALMBORG, B. NYSTRÖM AND L. SJÖBERG

Cultures from cleaned wash-basins in the Unit showed a reduction in the number of bacteria $5 \mathrm{hr}$ after cleaning. In some cases the cleaning entirely

Week

$\begin{array}{lllllllllllll}1 & 5 & 10 & 15 & 20 & 25 & 30 & 35 & 40 & 45 & 50 & 55 & 60\end{array}$

OPERATING THEATRE

EXAMINATION ROOM

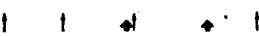

SLUICE ROOM

+4 t 111

$1+1$

NLIRSES'OFFICE

1

11111111

1

Days on which

environment was sampled

- Strain A

1 Other strains

FIG. 3.-Isolation of Pseudomonas aeruginosa from the environment of service rooms.

Growth

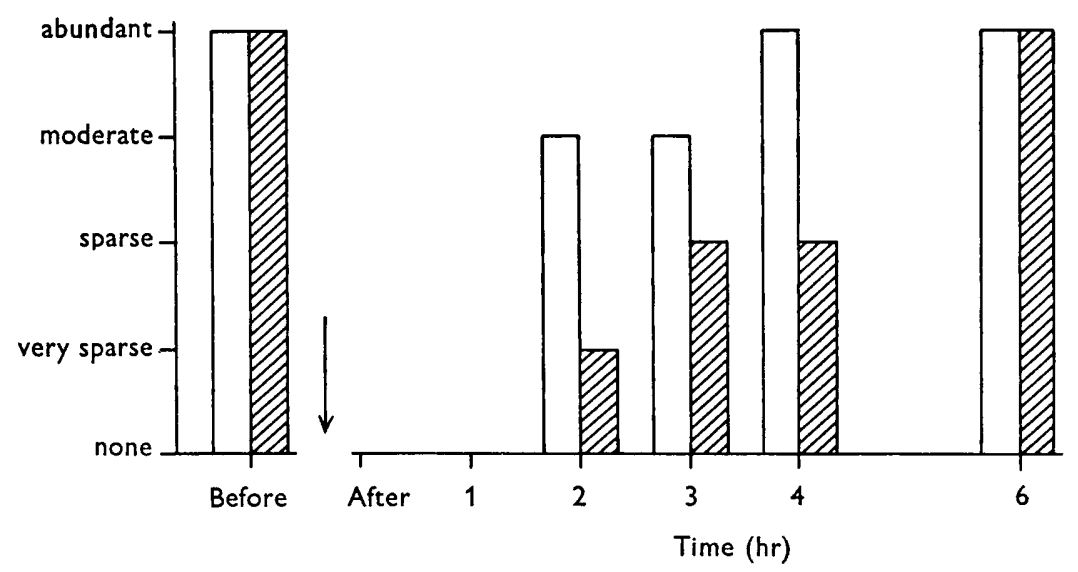

FIG. 4.-Isolation of Pseudomonas aeruginosa from a laboratory wash-basin before and at various times after disinfection (see Materials and methods). $\downarrow$ Application of disinfectant; $\square$ with flushing for $5 \mathrm{~min}$. in each $\mathrm{hr}$; without flushing.

removed the pseudomonas strain from the wash-basins. These effects, however, were only temporary; $24 \mathrm{hr}$ later the number of pseudomonas-colonised 
wash-basins and the quantity of pseudomonas in the colonised basins were the same as before cleaning.

\section{Discussion}

In this study, 45 of 73 patients (62 per cent.) were colonised with $P$. aeruginosa. This figure is similar to those reported by Birke et al. (1969) and by Wickman (1970) from the same Unit during earlier periods. The frequency of pseudomonas colonisation was higher the greater the extent of the burn.

According to conventional rules for the phage-typing of Staphylococcus aureus, a difference of at most two reactions is permitted between the typing patterns of pairs of isolates that are judged to belong to the same strain. This rule was followed also in the present study in interpreting the phage-typing patterns of $P$. aeruginosa. Further support for this procedure is that two of the phages in the commonest pattern, 21 and 68, produced microplaques. With this interpretation, 33 per cent. of the phage-typed pseudomonas isolates belong to the same strain. Only 2 per cent. of the isolates were untypable with the set of phages used. This is fewer than in the study by Sjöberg and Lindberg (1968) on a number of other clinical series. The result of phage-typing in this study was thought to provide so good an epidemiological guide that serological typing or pyocine typing was considered unnecessary.

The study shows that $P$. aeruginosa may be present for a long period of time in a ward environment without colonising the patients. Figs. 1 and 2 show several examples of this as regards strain A (e.g., rooms A, D, F, and G after the summer cleaning). This may be partly explained by the fact that the patients were kept in the rooms, and thus exposed to the strain, only for a short period. A further explanation may be also that some patients had only insignificant or nearly healed burns and therefore a fairly high resistance to colonisation with pseudomonas. This was the case, for example, in room A, where a superficial second-degree burn was treated in wk 23-30, in room D, where an almost healed third-degree burn was treated in wk 25-28, and in room G, where a superficial second-degree burn was treated in wk 27-30. But in room $\mathrm{D}$ a recent second-degree burn was treated during wk 22-24 and in room $\mathrm{G}$ a recent third-degree burn in wk 31-35 without being colonised with strain A, which was simultaneously present in the environment in these rooms. This may be explained as an effect of the therapy, with exposure treatment of the major burns in warm dry air, restriction of the use of antibiotics, and the routine of wearing gown and gloves by those who came into contact with the patients. These may also explain the remarkably low incidence of clinical infections with $P$. aeruginosa in the present series.

The attempts to decontaminate wash-basins and sinks were unsuccessful, though they may have caused temporary reduction in the number of bacteria present. This reduction, however, may also have been due to the fact that the frequent sampling from the environment led to a general tightening-up of the cleaning routines. When the study had been under way for a time, it was not uncommon to see the staff unscrew the water traps under the wash-basins to 
clean them inside, a procedure that had seldom formed part of the previous cleaning routine.

Thirty-three patients were examined once weekly for the presence of pseudomonas in faeces. In 17 of them (52 per cent.) $P$. aeruginosa was isolated from the faeces but in only three of them did it seem likely that the burn had been colonised from the intestinal flora. Sutter and Hurst (1966), who also used a phage-typing technique, studied 14 patients with burns colonised by $P$. aeruginosa and found the organism in the faeces of 12 of them, but concluded that only in a minority could the burn have been colonised from the intestinal flora. Kefalides et al. (1964) found $P$. aeruginosa in the faeces of less than onethird of burn patients. Shooter et al. (1966), who studied patients in a surgical ward, found that 38 per cent. of them carried $P$. aeruginosa in their intestinal flora at some time during their stay in the ward. The observed frequency of pseudomonas in the intestinal flora depends on the frequency of sampling and on the methods used for isolating the organism. Comparisons between different investigations must therefore be made with many reservations. In the present investigation, the observed frequency of intestinal carriage is higher than that reported by Kefalides $e t$ al. and by Shooter $e t$ al. Infection from the intestine to the burn was not commonly observed in our series or in that of Sutter and Hurst.

As expected, there seems to be no simple answer to the question of the routes of infection for $P$. aeruginosa in burns. Although in this study a single strain appeared to predominate in the Unit, no predominant route of infection within the Unit was observed.

\section{SUMMARY}

An epidemiological investigation of the spread of Pseudomonas aeruginosa in a burns unit was conducted by means of a phage-typing technique. During the period of treatment 62 per cent. of the patients were colonised with $P$. aeruginosa in their burns. The frequency of colonisation was correlated to the extent and depth of the burns. Thirty-three per cent. of the isolates of $P$. aeruginosa belonged to the same phage type, 1214/109/F8.

Endogenous infection from intestinal flora to burn in the same patient was not commonly observed. In several cases $P$. aeruginosa was present for a long period of time in the ward environment without colonising patients being treated for open, extensive and recent burns. The reason for this is discussed. No predominant route of infection could be demonstrated.

It was not possible permanently to decontaminate the environment by disinfection of wash-basins and sinks.

\section{REFERENCES}

Altemeier, W. A., Todd, J. C., AND Inge, W. W. 1966. Newer aspects of septicemia in surgical patients. Archs. Surg., Chicago, 92, 566.

BARCLAY, T. L., AND DeXTER, F. 1968. Infection and cross-infection in a new burns centre. Br. J. Surg., 55, 197.

Barr, P.-O., Birke, G., Liljedahl, S.-O., and Plantin, L.-O. 1967. Treatment of burns with warm dry air. Lancet, 1, 1276. 
Birke, G., LilJedahl, S.-O., Bäckdahl, M., and NyléN, B. 1964. Studies on burns. VIII. Analysis of mortality and length of hospital care for 603 burned patients referred for primary treatment. Acta chir. scand., Suppl. 337.

Birke, G., LilJedAHL, S.-O., AND Norberg, R. 1969. Studies on burns. XI. Immunoglobulin and $\alpha$ macroglobulin patterns and bacterial flora during treatment of burns with warm dry air. Scand. J. Plast. Reconstr. Surg., 3, 39.

Graber, C. D., Latta, Ruth, Vogel, E. H., JR, and Brame, R. 1962. Bacteriophage grouping of Pseudomonas aeruginosa: with special emphasis on lysotypes occurring in infected burns. Amer. J. Clin. Path., 37, 54.

Kefalides, N. A., Arana, J. A., Bazan, A., Velarde, N., and Rosenthal, S. M. 1964. Evaluation of antibiotic prophylaxis and gamma-globulin, plasma, albumin and salinesolution therapy in severe burns; bacteriologic and immunologic studies. Ann. Surg., 159, 496.

King, Elizabeth O., Ward, Martha K., and Raney, D. E. 1954. Two simple media for the demonstration of pyocyanin and fluorescin. J. Lab. Clin. Med., 44, 301.

LiLJEDAHL, S.-O. 1970. The treatment of burns. Opusc. Med., 15, 179 (in Swedish).

Lindberg, R. B., Latta, R. L., Brame, R. E., AND Moncrief, J. A. 1964. Definitive bacteriophage typing system for Pseudomonas aeruginosa. Bact. Proc., 81.

Lowbury, E. J. L. 1970. Controlled trials of patient-isolation in a burns unit. In: Aerobiology. Proceedings of the third international symposium, 1969, edited by I. H. Silver, London and New York, p. 131.

Lowbury, E. J. L., and Fox, Jean 1954. The epidemiology of infection with Pseudomonas pyocyanea in a burns unit. J. Hyg., Camb., 52, 403.

MacMillan, B. G. 1965. Local care and infection in burns. J. Trauma, 5, 292.

Moncrief, J. A., AND Teplitz, C. 1964. Changing concepts in burn sepsis. J. Trauma, $4,233$.

MüLLER, F. E. 1967. Gentamicin in the treatment of pseudomonas infections complicating burns. In Gentamicin, First International Symposium, Paris, January 1967, Basel, p. 148.

Shooter, R. A., Walker, K. A., Williams, Veronica R., Horgan, Gillian M., Parker, M. T., Asheshov, Elizabeth H., AND Bullimore, Juliet F. 1966. Faecal carriage of Pseudomonas aeruginosa in hospital patients; possible spread from patient to patient. Lancet, 2, 1331.

Sjöberg, L., AND LindBerg, A. A. 1968. Phage typing of Pseudomonas aeruginosa. Acta path. microbiol. scand., 74, 61.

SUtTer, Vera L., AND HuRst, VALerie 1966. Sources of Pseudomonas aeruginosa infection in burns: study of wound and rectal cultures with phage typing. Ann. Surg., 163, 597.

Sutter, Vera L., Hurst, VAlerie, and FenNell, J. 1965. A standardized system for phage typing Pseudomonas aeruginosa. Hlth Lab. Sci., $2,7$.

WICKMAN, KRISTINA 1970. Burns, bacteria and antibiotics. Försvarsmedicin, 6, 201. 